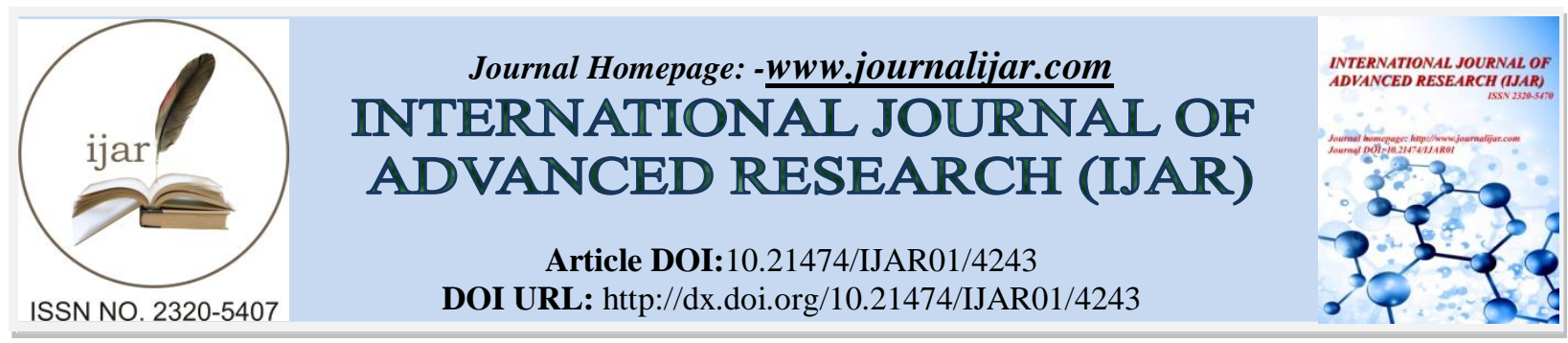

RESEARCH ARTICLE

\title{
ELECTRICAL CONDUCTIVITIES OF SYNTHESIZED POLY o- PHENYLENEDIAMINE AND ITS NANOCOMPOSITES.
}

J. Deepa and *Jaya Shanthi R.

PG \& Research Department of Chemistry, Auxilium College, Vellore, Tamil Nadu, India.

\section{Manuscript Info}

Manuscript History

Received: 23 March 2017

Final Accepted: 25 April 2017

Published: May 2017

Key words:-

Poly (o-Phenylenediamine),

Nanocomposites, Thermal property,

Electrical conductivity

\section{Abstract}

Poly (o-Phenylenediamine) and their metal oxide nanocomposites using different concentrations like 5\%, 10\% and $15 \%$ of $\mathrm{SiO}_{2}$ nanoparticles were synthesized by chemical oxidative polymerization method using ammonium persulphate as an oxidant in the presence of $\mathrm{HCl}$. The formation of polymers and their nanocomposites were confirmed from the UV-Vis and FT - IR spectroscopy. The formation of Poly (o-Phenylenediamine) nanocomposites were confirmed by the change of polymer colour from red to brown and found to exhibit band at $446 \mathrm{~nm}$ in UV-visible spectroscopy. The crystalline nature of the synthesized polymers and their nanocomposites were determined from the XRD studies. The SEM images of the polymers recorded at different magnification shows rod like structure and found to change to flake like structures in the polymer nanocomposites synthesized at different concentration of $\mathrm{SiO}_{2}$ nanoparticles. The TEM recorded at different angle confirms the core shell structures. The stability of the synthesized polymer and its nanocomposites were substantiated from thermal studies carried out using TGA, DTA and DSC. The comparative electrical conductivities of the polymer and its nanocomposites shows that the polymer nanocomposites exhibit higher conductivities compared to the polymer and the electrical conductivity was found to be higher for the polymer nanocomposite synthesized with $15 \%$ of $\mathrm{SiO}_{2}$. The polymer and its nanocomposites show semiconducting nature.

Copy Right, IJAR, 2017,. All rights reserved.

\section{Introduction:-}

Conducting polymers consisting of conjugated electronic structures have received considerable attention in the field of material science due its promising technological applications ${ }^{1}$. Among conducting polymers; polyaniline and its derivatives have attracted much attention due to its ease of synthesis ${ }^{2-4}$. It has been demonstrated that organic compounds, in particular polymers have many interesting properties such as solubility, procesability, environmental stability, and electroactivity ${ }^{5}$. Nanocomposites are generally defined as composites in which the components have at least one dimension (i.e., length, width or thickness) in the size range of 1-100 $\mathrm{nm}^{6}$. Nanocomposites differ from traditional composites in a sense that interesting properties can result from the complex interaction of the nanostructured heterogeneous phases. In addition, nanoscopic particles of a material differ greatly in the analogous properties from a macroscopic sample of the same material ${ }^{7}$.Nanocomposites containing organic polymers and 
inorganic particles provide a completely new class of materials with novel properties ${ }^{8} . \mathrm{SiO}_{2}$ is an excellent catalyst support because of its chemical inertia, thermal stability and adsorption of reactants ${ }^{9}$. Silicon oxide is normally generated from sand that is extracted after a fusion of high temperature. Characterisation temperature was determined via the temperature at which the highest Specific Surface Area (SSA) and highest amount of silica were observed which was $700^{\circ} \mathrm{C}^{10}$.

The polymer/silica nanocomposites can improve the physical properties such as the mechanical and thermal properties of the materials and also exhibit some unique properties ${ }^{11}$. Recently, several groups have combined conductive polymers with metal oxides to generate hybrid composites that possess higher reversible capacity, redox cyclability and structural stability. The properties of composites ofsuch kind are strongly dependent on concentration of polymer ${ }^{12-16}$. An important attractive property of electroactive polymers are in fields of Light Emitting Diodes (LEDs) and solar cells (SC), field effect transistors, photovoltaic cells, and lasers etc ${ }^{17}$.

In the present study, Poly (o-Phenylenediamine) (PoPDA) and its nanocomposites were synthesized by chemical oxidative polymerization with different concentrations of $\mathrm{SiO}_{2}$ nanoparticle. The synthesized polymer and its nanocomosites were characterized and studied for their conducting properties.

\section{Materials and Methods:- \\ Materials:-}

Monomer Poly (o-Phenylenediamine) (PoPDA), Ammonium persulphate (APS, 99.99\%), silicon dioxide are product of sigma- Aldrich and Hydrochloric acid were procured from Merck Ltd., India. All chemicals were of analytical reagent grade and were used as received without any further purification in the present study.

\section{Methods:-}

\section{Synthesis of Poly o-Phenylenediamine:-}

The Poly o-Phenylenediamine and nanocomposites were synthesized by chemical oxidative polymerization technique using a standard procedure with slight modification ${ }^{18}$, ${ }^{19}$. The precipitated polymer was washed with distilled water until the filtrate was colourless then with acetone and methanol to remove excess initiator, monomer and oligomers. Finally the resultant polymer precipitate was dried at room temperature for 24 hours.

\section{Characterization techniques:-}

Fourier transform infrared of the synthesized samples were performed using ABB-MB-3000 spectrometer in $\mathrm{KBr}$ medium at room temperature. UV-Vis spectra were recorded on a Perkin Elmer Lamba spectrophotometer by dissolving the polymers in DMSO as a solvent. The X-ray powder diffraction patterns were recorded on a panalytical diffractometer $(\mathrm{Cuk} \alpha)$ radiation source. The XRD measurements were carried out by applying a step scanning method ( $2 \theta$ ranged from $0-70^{\circ}$ ) with the scanning speed of $4^{\circ} \mathrm{min}^{-1}$. The morphological study of the synthesized polymers was carried out using Scanning Electron Microscopy (SEM model: Jeol 6390 LV) with accelerating voltage of $0.5 \mathrm{kV}$ to $30 \mathrm{kV}$. Transmission electron microscopy (TEM model Tecnai T20 G2 S-TWIN) was used to investigate the dispersion of PoPDA/ $\mathrm{SiO}_{2}$ composites. TGA/DTA was recorded using Perkin Elmer Diamond under a nitrogen atmosphere up to $700^{\circ} \mathrm{C}$ using a heating rate of $10^{\circ} \mathrm{C} / \mathrm{min}$. Differential scanning calorimetry (DSC) was measured using Mettler Toledo DSC $822^{\mathrm{e}}$ from room temperature to $500^{\circ} \mathrm{C}$. The electrical conductivity was measured at room temperature by the four-point probe technique using LCR meter HP484A.

\section{Results and Discussion:-}

FT-IR spectroscopy:-

FT-IR spectrum of the PoPDA and PoPDA synthesized with $5 \%, 10 \%$ and $15 \%$ of $\mathrm{SiO}_{2}$ are given figure 1 . The single peaks at $3227 \mathrm{~cm}^{-1}$ is due to the $\mathrm{N}-\mathrm{H}$ stretching vibrations of the $-\mathrm{NH}-$ group $^{20}$. The two peaks at 3380 and $2953 \mathrm{~cm}^{-1}$ are associated with the asymmetrical and symmetrical $\mathrm{N}-\mathrm{H}$ stretching vibrations of the $\mathrm{NH}_{2}$ group respectively. Two strong peaks at $1629 \mathrm{~cm}^{-1}$ and $1531 \mathrm{~cm}^{-1}$ are assigned to the $\mathrm{C}=\mathrm{C}$ and $\mathrm{C}=\mathrm{N}$ group in phenazine ring $^{21}$. The peak at $1359 \mathrm{~cm}^{-1}$ and $1286 \mathrm{~cm}^{-1}$ are associated with C-N-C stretching in the benzenoid and quinoid rings ${ }^{22}$. Furthermore, the bands at $718 \mathrm{~cm}^{-1}$ and $609 \mathrm{~cm}^{-1}$ are the characteristic of C-H out- of- plane bending vibrations of benzene nuclei in the phenazine skeleton ${ }^{23}$. However with addition of $\mathrm{SiO}_{2}$ nanoparticles, the characteristic peaks are slightly shifted to the lower wave numbers with slight increase in their intensities. The shifts in the frequencies are due to the interaction between the $\mathrm{SiO}_{2}$ nanoparticles and polymer chain which affect the 
electron densities and bond energies ${ }^{24}$. A strong absorption band at 1073 and $804 \mathrm{~cm}^{-1}$ are due to the stretching and bending vibration of $\mathrm{SiO}_{2}$ present in the polymeric backbone ${ }^{25}$.

\section{UV-Vis spectroscopy:-}

The UV- Vis spectra of PoPDA and PoPDA/SiO ${ }_{2}$ nanocomposites with different wt $\%$ of $\mathrm{SiO}_{2}$ nanoparticles $(5,10$ and $15 \%$ ) are given in figure 2. The UV-Vis absorption spectra of PoPDA reveal that the absorption maxima at 452 and $316 \mathrm{~nm}$ are originating from the charge transfer-excitation. The PoPDA/SiO ${ }_{2}$ nanocomposites exhibited two major peaks at 280 and $446 \mathrm{~nm}$. The peak at $280 \mathrm{~nm}$ is assigned to $\pi-\pi^{*}$ transition of the benzenoid and quinoid ring and the peak at $446 \mathrm{~nm}$ is due to $\pi-\pi^{*}$ transition associated with the phenazine ring conjugated to the lone pair of electrons present on the nitrogen of the $\mathrm{NH}_{2}$ groups. The comparative study of the UV shows that the polymers nanocomposites are slightly shifted to lower wave length compared to the polymer ${ }^{26}$.

\section{X- Ray Diffraction:-}

The X-ray diffraction pattern of PoPDA shows two sharp peaks at $2 \theta=24.95$ and 25.59. The inter-planar distances are found to be $40.95 \mathrm{~nm}$ and $38.74 \mathrm{~nm}$ and this is responsible for the crystalline nature of the polymer as shown figure 3. The average crystalline size is calculated using Debye - Scherer equation $D=[0.89] /[\beta \cos \theta]$, Where $\beta$ is full width of half maxima and the crystalline size of the polymer is found to be $8.340 \mathrm{~nm}$. Generally, polymer is considered to be amorphous but here the synthesized Phenylenediamine polymer is showing crystalline structure due to Phenylenediamine and planar nature of benzenoid and quinoid structure. The XRD studies of the synthesized polymer nanocomposites are found to be amorphous in nature ${ }^{27}$.

\section{Scanning Electron Microscopy:-}

The SEM images of PoPDA and PoPDA/SiO 2 nanocomposites are given in figures 4a-d. Figure 4a shows the SEM image of PoPDA which is found highly rod like structure. The SEM morphology of polymer nanocomposites with $5 \%, 10 \%$ and $15 \%$ of $\mathrm{SiO}_{2}$ nanoparticles are found to be highly agglomerated and granular in shape (4b), spherical in shape (4c) which decreases the intragranular distance as the concentration of $\mathrm{SiO}_{2}$ increases in PoPDA and with $15 \%$ of $\mathrm{SiO}_{2}$ (fig.4d), the SEM image with further increases in weight percentage of metal oxide particles a transition from spherical to flake like structure ${ }^{28}$.

\section{Thermogravimetric Analysis:-}

Thermogravimetric analysis (TGA) is used to study the thermal stability of PoPDA and PoPDA/SiO 2 synthesized at different concentration of $\mathrm{SiO}_{2}$ nanoparticles like 5, 10 and $15 \%$. The polymer and its nanocomposites are found to have three stages of thermal transition as shown in figure 5 and the thermal degradation of the polymer and its nanocomposites are almost similar. The first thermal transition occurred at $128^{\circ} \mathrm{C}$ is mainly due to the removal of moisture ${ }^{29}$. The second thermal transition at $287^{\circ} \mathrm{C}$ may be attributed to the removal of dopant ${ }^{30}$ and the final weight loss at $483^{\circ} \mathrm{C}-700^{\circ} \mathrm{C}$ correspond to the degradation of the polymer ${ }^{31}$. The total residue was found to be $39.12 \%$ (wt $\%$ ) for PoPDA and for the polymer nanocomposites the total residues are found to increase slightly to $42.05 \%$, $43.85 \%$ and $45.78 \%$ (wt \%) with $5 \%, 10 \%$ and $15 \% \mathrm{SiO}_{2}$ nanoparticles after degradation.

\section{Differential Scanning Calorimetry:-}

The DSC of PoPDA and PoPDA/5\% $\mathrm{SiO}_{2}, \mathrm{PoPDA} / 10 \% \mathrm{SiO}_{2}$ and PoPDA $/ 15 \% \mathrm{SiO}_{2}$ are given figure 6 and it shows an endothermic peak at $96.47^{\circ} \mathrm{C}$ which is due to the glass transition temperature (Tg). The polymer starts to melt are shown by endothermic peak at $370^{\circ} \mathrm{C}$ which is characteristics of melting temperature ${ }^{32}$.

\section{Transmission Electron Microscopy:-}

Transmission Electron Microscopy images of polymer nanocomposites with 15\% of SiO2 nanoparticle at different angles are given in figure 7 and it found to have aggregated spherical like morphology. The TEM micrograph shows that the particle size of synthesized polymer nanocomposites range from $40-45 \mathrm{~nm}$. Moreover the outer shell of the TEM image of the outer core of the particle is more bright compared with the dark inner core and this forms the core- shell feature of the PoPDA/SiO $/ 2$ nanocomposites ${ }^{33}$. The formation of $\mathrm{PoPDA} / \mathrm{SiO}_{2}$ core shell nanocomposites was attributed to the strong electrostatic interaction between PoPDA and $\mathrm{SiO}_{2}$.

\section{Electrical conductivity:-}

The electrical conductivity of PoPDA and PoPDA $/ \mathrm{SiO}_{2}(5 \mathrm{wt} \%, 10 \mathrm{wt} \%$ and $15 \mathrm{wt} \%)$ were determined using fourpoint-probe technique at room temperature and the results are given in figure 8 . The conductivity values of PoPDA and its nanocomposites were evaluated using bulk resistance $\left(\mathrm{R}_{\mathrm{b}}\right)$ using the formula: $\quad \sigma=(\mathrm{t} / \mathrm{A})(1 / \mathrm{Rb}) \mathrm{S} / \mathrm{cm}$, 
Where, $t$ is the thickness of the pellet, $A$ is the area of pellet and $R_{b}$ is the bulk resistance of the pellet. The electrical conductivity of the PoPDA and PoPDA synthesized with 5, 10, and $15 \% \mathrm{SiO}_{2}$ nanoparticles are found to be 1.79 $\times 10^{-8} \mathrm{~S} / \mathrm{cm}, 1.59 \times 10^{-7}, 2.13 \times 10^{-7}$ and $2.78 \times 10^{-7} \mathrm{~S} / \mathrm{cm}$. The results clearly show that the conductivity increases from polymer to the polymer nanocomposites and as the concentration of the $\mathrm{SiO}_{2}$ increases, the conductivity increases slightly. When added different concentration of metal oxide nanopowder, it was found that the electrical conductivity of polymer $\mathrm{SiO}_{2}$ nanocomposites was found to improve ${ }^{34}$. The electrical conductivity of the polymer nanoparticle increase when the polymer was developed with metal oxide used.

Fig 1:- FT-IR spectra of PoPDA and its $\mathrm{SiO}_{2}$ nanoparticles at different concentrations

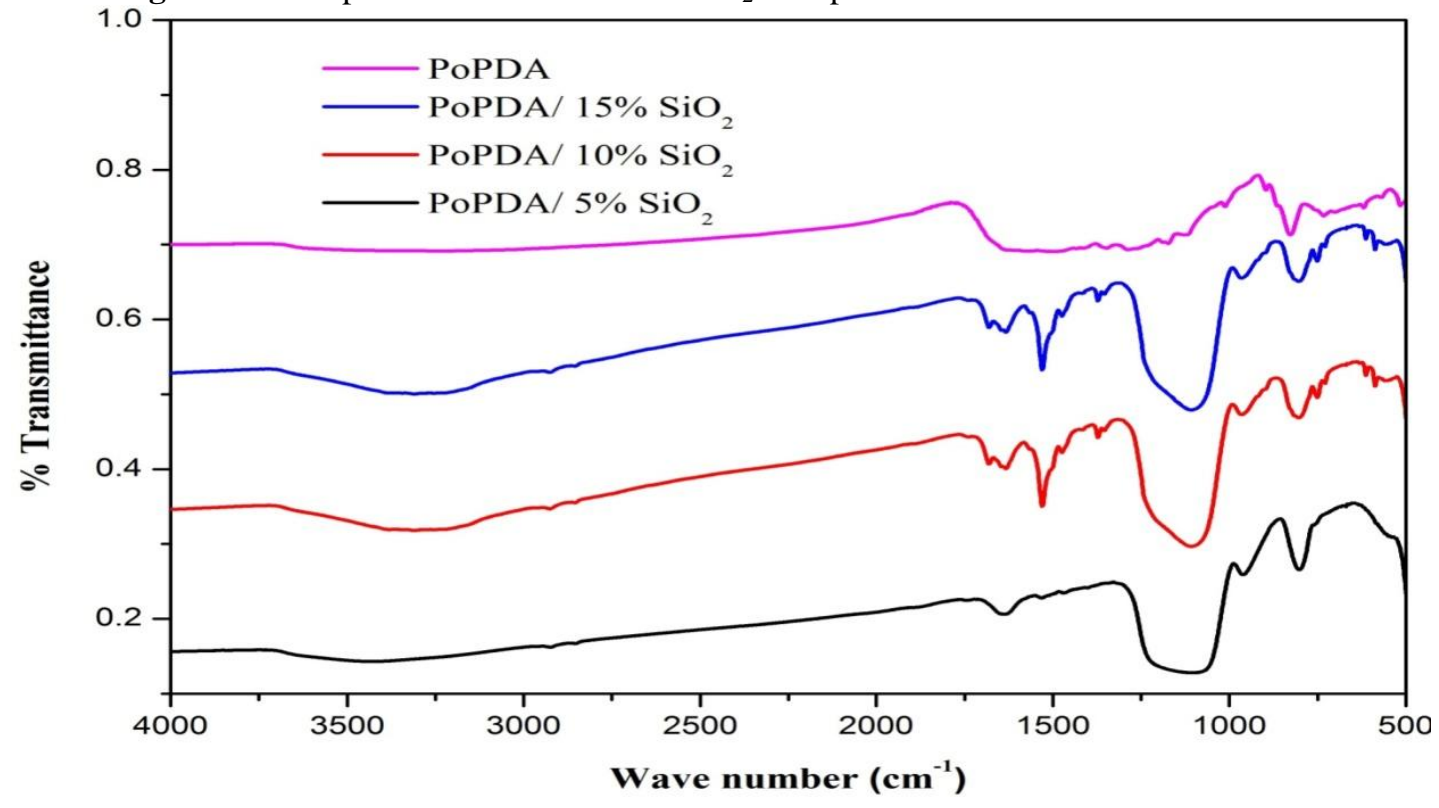

Fig 2:- UV-Vis spectra of PoPDA and its $\mathrm{SiO}_{2}$ nanoparticles at different concentrations.

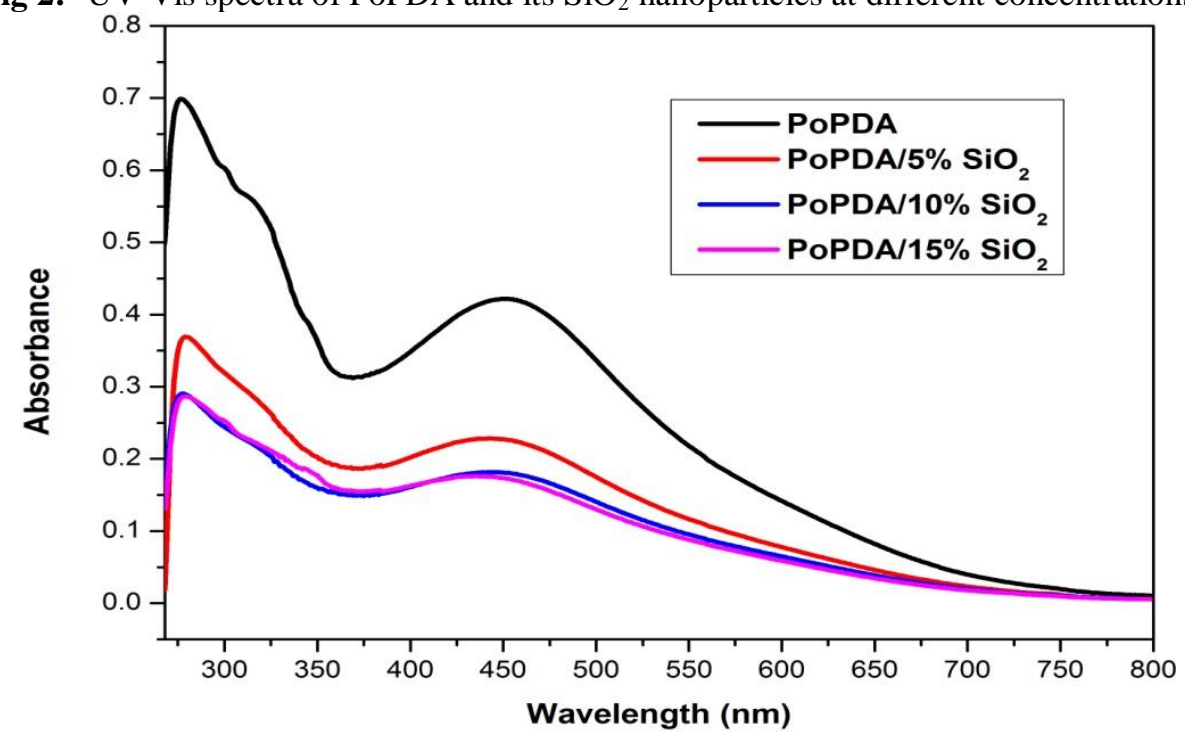


Fig 3:- XRD spectra of PoPDA and its $\mathrm{SiO}_{2}$ nanoparticles at different concentrations

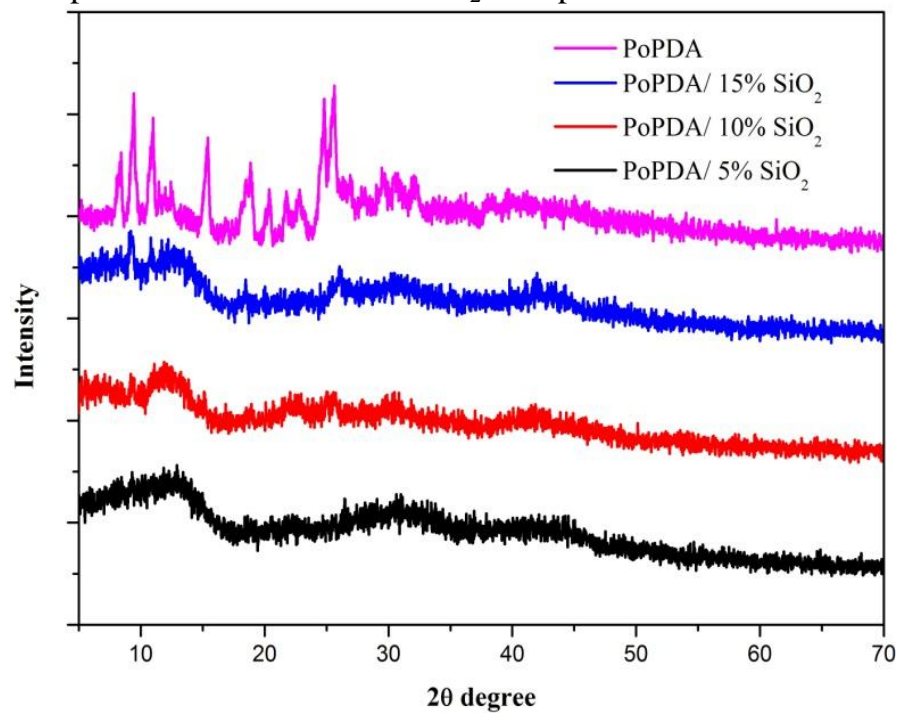

Fig 4:- SEM image of PoPDA and its $\mathrm{SiO}_{2}$ nanoparticles at different concentrations.
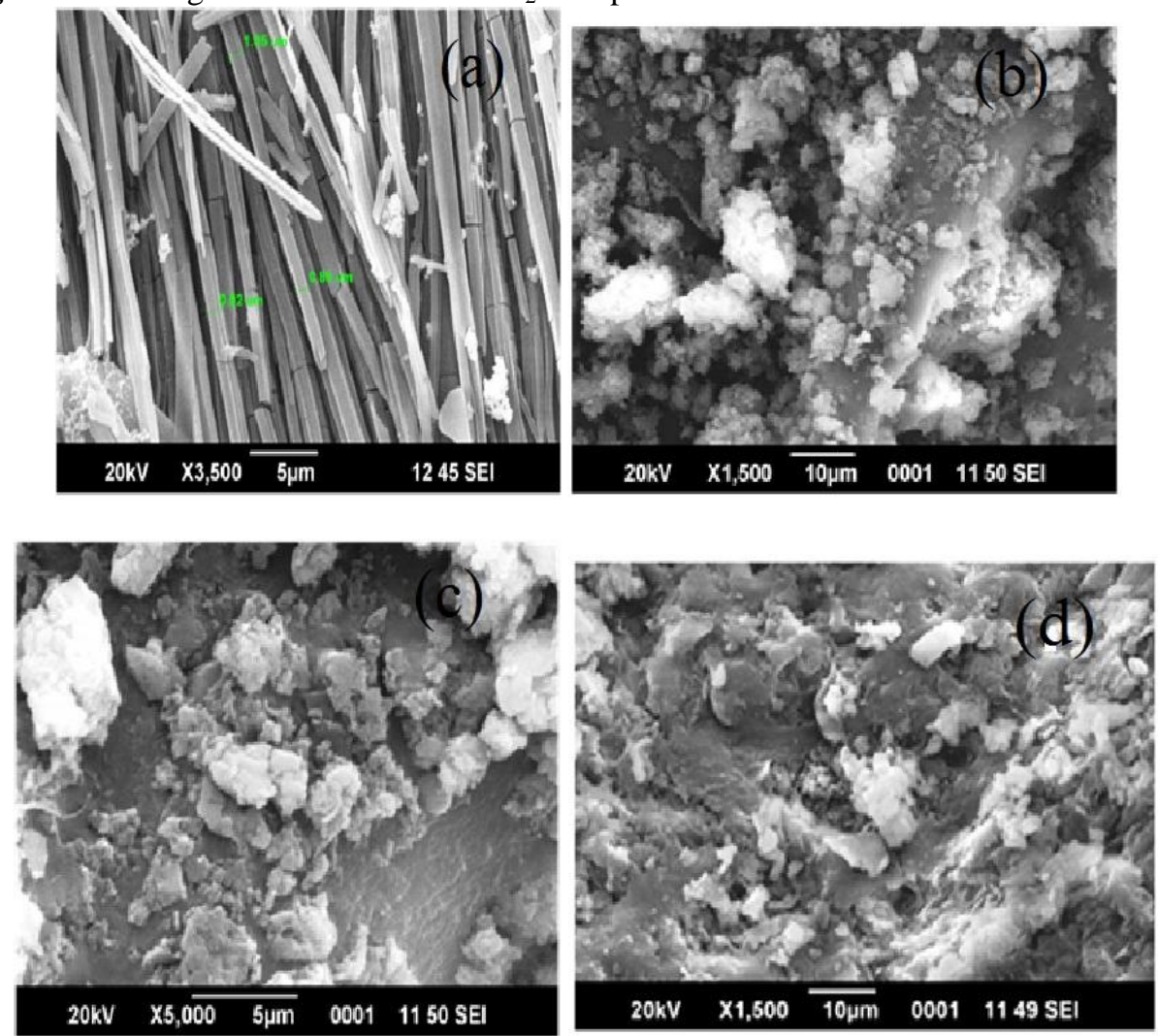
Fig 5:-TGA of PoPDA and its $\mathrm{SiO}_{2}$ nanoparticles at different concentrations.

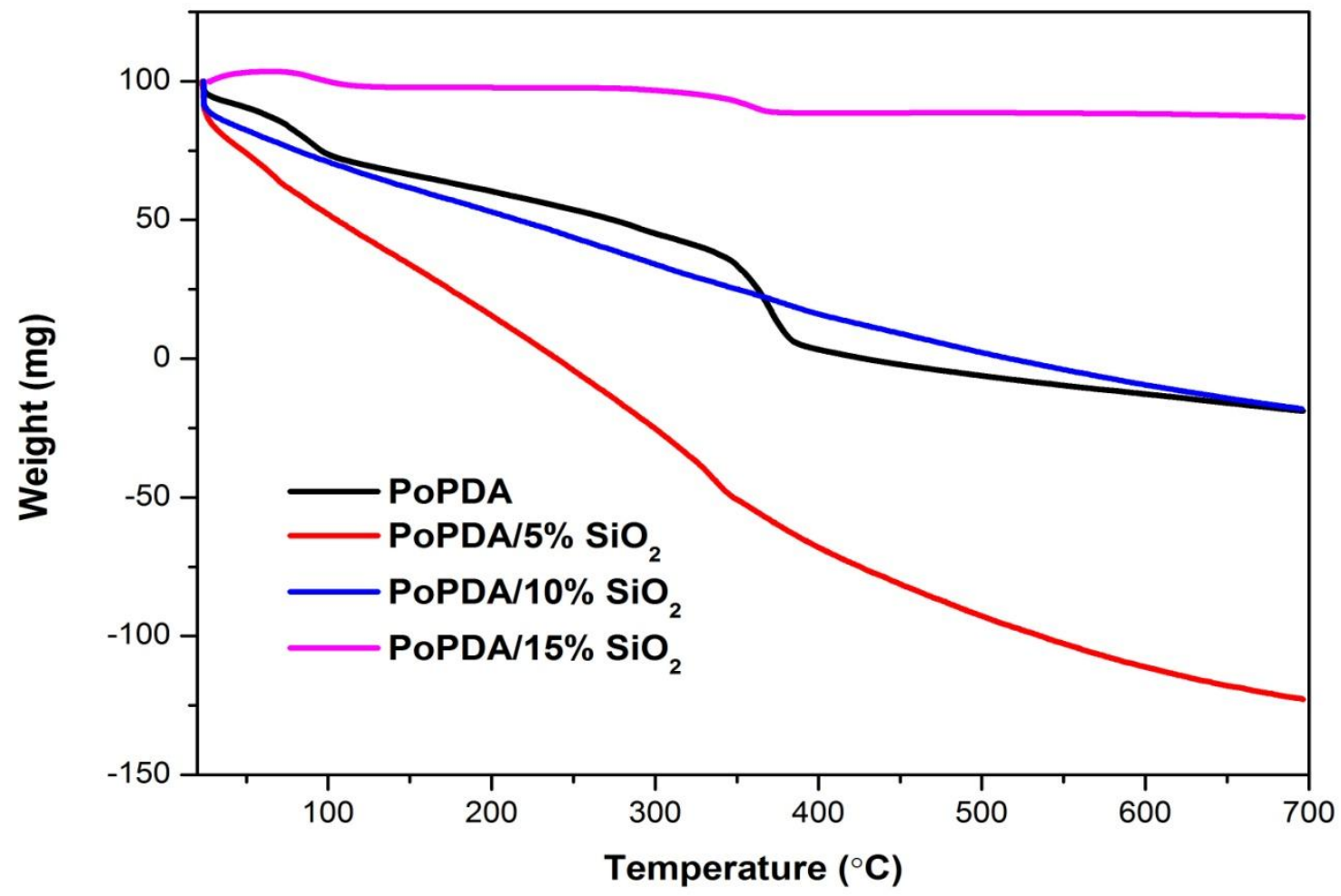

Fig 6:- DSC of PoPDA and its $\mathrm{SiO}_{2}$ nanoparticles at different concentrations

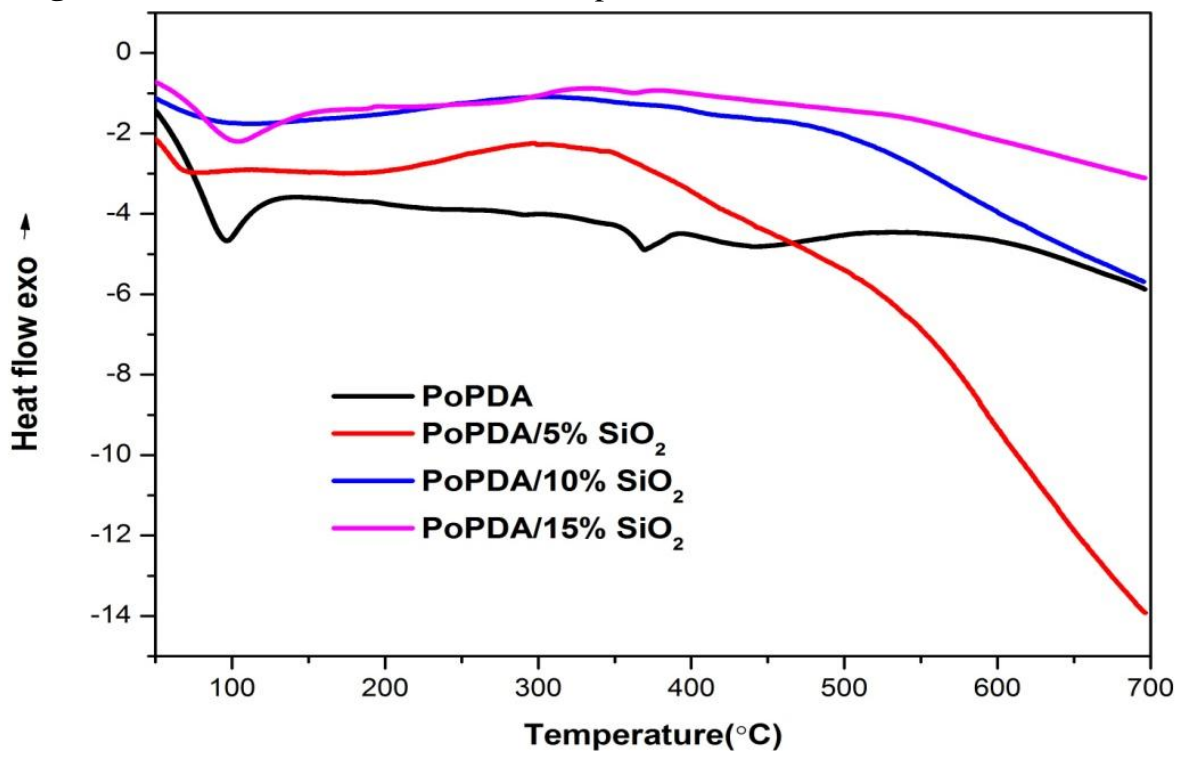


Fig 7:-TEM image of PoPDA/ $15 \% \mathrm{SiO}_{2}$ nanocomposites at different angles

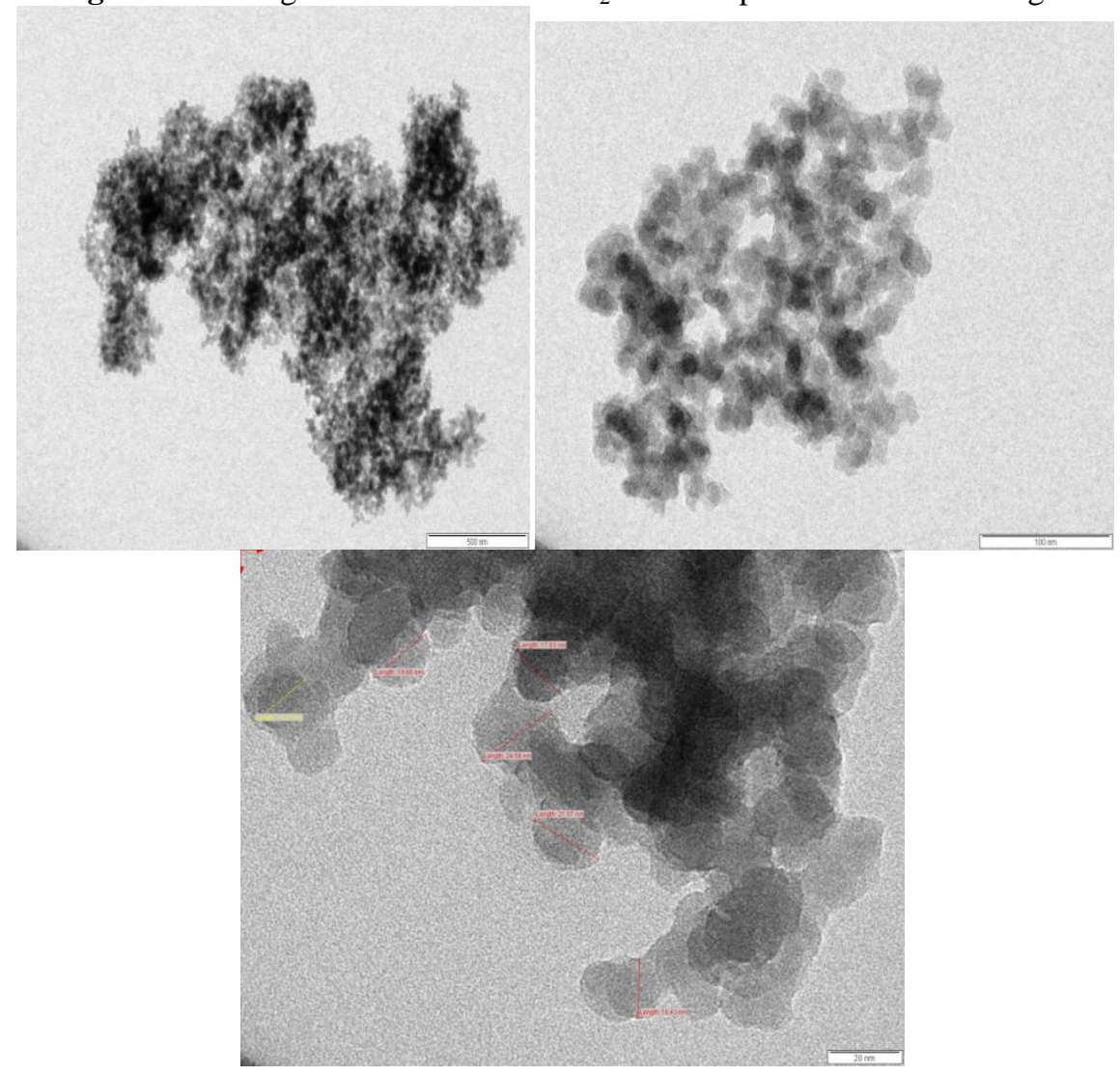

Fig 8:-Eectrical conductivity of PoPDA and its $\mathrm{SiO}_{2}$ nanoparticles at different concentrations

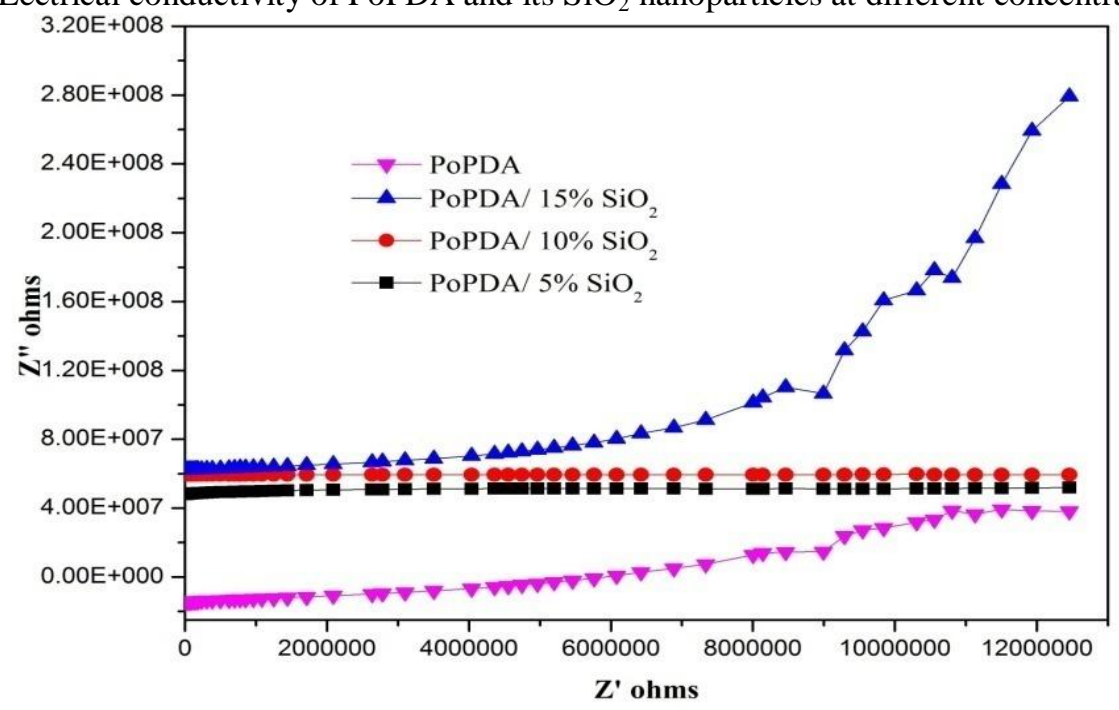

\section{Conclusion:-}

The PoPDA and PoPDA $/ \mathrm{SiO}_{2}$ nanocomposites have been successively prepared via chemical oxidative polymerization method. The synthesized polymer and nanocomposites were characterized using FT-IR, UV-Vis spectroscopy and it is confirmed the formation of the polymers. The morphology of the polymer and its nanocomposites studied by SEM shows different morphology depending on the polymer and its nanocomposites. The XRD studies confimed that the synthesized nanocomposites were amorphous in nature unlike the polymer. The formation of core shell type morphology of $\mathrm{SiO}_{2}$ nanoparticles was confirmed from TEM analysis. The thermal stabilities of the polymers and its composites were confirmed from the TGA/DAT and DSC studies. The 
conductivity studies of the polymer and its nanocomposites of PoPDA shows semiconducting in nature and the conductivity is higher in polymer nanocomposites than the polymer.

\section{Reference:-}

1. Anoop Kumar S, Hema Bhandari, Chandrica Sharma, Fehmeed Khatoon and Sundeep K Dhawan, Poly Int., 2012, 11, 822

2. Borole DD, Kapadi UR, Mahulikar PP, Hundiwale DG, J Mater Sci, 2006, 41, 1983-1990.

3. Camatel JL, Lacroix JC, Aciyach S, Chaneching K, Lacaze PC, Synth Met.,1998 93: 133.

4. Liao DC, Hsich KH, Chern YC, Ho K S, Synth Met., 1997, 87, 61.

5. Huseyin Zengina and Belgin Erkan, Polym. Adv. Technol., 2010, 21, 216

6. Paulo Meneghetti, Syed Qutubuddin, ThermochimicaActa., 2006, 442,74

7. Prasanna Kumar Sahoo, Dillip Kumar Behera, Matru Prasad Dash, Bijaya Kumar Parija, Munesh Chandra Adhikari and Nayak PL, The International Journal of Engineering And Science., 2013, 2(1), 141-148

8. Dutta K, De SK, Physics Letters A., 2007, 361, 141

9. Zhenyan Liu, Yue-E. Miao, Mingkai Liu, Qianwei Ding, Weng Weei Tjiu, Xiaoli Cui, Tianxi Liu, Journal of Colloid and Interface Science., 2014, 424, 49-55

10. Olawale O, Akinmoladun, Oyawale AI, And Atiko Rejoice FA, International Journal of Scientific \& Engineering Research., 2013, 4(2), 2229

11. Iric-Marjanovic G C, Dragic Evic, Milojevic M, Mojovic M, Mentus S, Dojic Inovic B, Marjanovia B, Stejskal J, J.phys.chem.B., 2009, 113, 7116

12. Asif Ali Khan, Leena Paquiza, Journal of Industrial and Engineering Chemistry., 2015,22, 208

13. Buttry D A, Langmuir., 1999, 15, 669

14. P. Gomez-Romero, J. Electrochem. Soc., 1999, 146, 29

15. Gambardella M T D, Torresi R.M, DeTorresi S.I, Buttry D.A, J. Electrochem. Soc., 2000, 147, 2437

16. E. Ruckenstein, Langmuir., 2002, 18, 6956

17. Hoppe H, Arnold N, Meissner D, Sariciftci N. S, Thin Solid Films., 2004, 451, 589

18. Aashis S. Roy, Koppalkar R, Anilkumar and Ambika Prasad V N, Ferroelectric., 2011, 413, 279

19. Sharanabasamma M Ambalgi, Hajeebaba K Inamdar, Manjula V T, Sannakki Nagaraja, Shrishail G Hogade, and Basavaraja Sannakki, International Journal of Engineering Research.,2016, 5(2), 119

20. Dharkar K P, Khamborkar A K and Kalambe A B., Research Journal of Chemical Sciences., 2012, 2(12), 11

21. Liang Wang, Shaojun Guo, Shaojun Dong, Materials Letters., 2008, 62, 3240

22. Qingli Hao, Baoming Sun, Xujie Yang, Lude Lu, Xin Wang, Materials Letters., 2009, 63, 334

23. X.G. Li, M.R. Huang, W. Duan, Chem. Rev.,2002, 102, 2925.

24. Melaku Wondwossen, Yadav OP and Kebede Tesfahun, Science, Technology and Arts Research Journal., 2014, 2305

25. Bange J P, Patil L S, and Gautam D K, Progress In Electromagnetics Research., 2008, 3, 165

26. Archana S, Jaya Shanthi R, Indian Journal of Advances in Chemical Science., 2013, 2(1), 83

27. Kavitha B, Sivakumar K and Narsimlu N, Indian Journal of Pure and Applied Physics., 2013, 51, 207

28. Mohana Lakshmi, Aashish S Roy, Syed Khasim, Muhammad Faisal, Sajjan K C and Revanasiddappa M, AIP Advances., 2013, 3(11), 2158

29. Mona H Abel Rehim, Nahla Ismail, Abd El- Rahman A A Badawy, and Gamal Turky, Journal of Materials Chemistry and Physics., 2011, 128, 507

30. Olad A, Nosrati R, Prog. Org. Coat., 2012, 68, 319

31. Muthirulan P, Rajendran N, Surface \& Coatings Technology., 2012, 206, 2072

32. Alan Riga, Ricardo Collins, Gregory Mlachak, Thermochimia Acta, 1998, 324, 135

33. Deivanayaki S, Ponnuswamy V, Mariappan R, Jayamurugan P, Optik., 2012, 124, 1089

34. Azza M. Mazrouaa, Yahia Abed M, Mansour NA and Mohamed MG, J Material Sci Eng., 2012,1(1), 103. 